\title{
Complementarity of neutron reflectometry and ellipsometry for the study of atmospheric reactions at the air-water interface
}

Article

Accepted Version

Sebastiani, F., Campbell, R. A. and Pfrang, C. (2015) Complementarity of neutron reflectometry and ellipsometry for the study of atmospheric reactions at the air-water interface. RSC Advances, 5 (129). pp. 107105-107111. ISSN 2046-2069 doi: https://doi.org/10.1039/c5ra22725a Available at https://centaur.reading.ac.uk/55428/

It is advisable to refer to the publisher's version if you intend to cite from the work. See Guidance on citing.

To link to this article DOI: http://dx.doi.org/10.1039/c5ra22725a

Publisher: Royal Society of Chemistry

All outputs in CentAUR are protected by Intellectual Property Rights law, including copyright law. Copyright and IPR is retained by the creators or other copyright holders. Terms and conditions for use of this material are defined in the End User Agreement. 


\section{CentAUR}

Central Archive at the University of Reading

Reading's research outputs online 


\title{
1 Complementarity of neutron reflectometry and ellipsometry for the study
}

2 of atmospheric reactions at the air-water interface.

3 Federica Sebastiani ${ }^{\mathrm{a}, \mathrm{b}}$, Richard A. Campbell ${ }^{\mathrm{b}^{*}}$ and Christian Pfrang. ${ }^{\mathrm{a}^{*}}$

${ }^{a}$ Department of Chemistry, University of Reading, P.O. Box 224, RG6 6AD, Reading, UK

${ }^{b}$ Institut Laue-Langevin, 71 avenue des Martyrs, CS 20156, 38042 Grenoble Cedex 9, France

* corresponding authors: campbell@ill.eu and c.pfrang@reading.ac.uk

\begin{abstract}
The combined application of neutron reflectometry (NR) and ellipsometry to determine the oxidation kinetics of organic monolayers at the air-water interface is described for the first time. This advance was possible thanks to a new miniaturised reaction chamber that is compatible with the two techniques and has controlled gas delivery. The rate coefficient for the oxidation of methyl oleate monolayers by gas-phase $\mathrm{O}_{3}$ determined using NR is $(5.4 \pm$ $0.6) \times 10^{-10} \mathrm{~cm}^{2}$ molecule $\mathrm{s}^{-1}$, which is consistent with the value reported in the literature but is now better constrained. This highlights the potential for the study of faster atmospheric reactions in future studies. The rate coefficient determined using ellipsometry is $(5.0 \pm 0.9) \times$ $10^{-10} \mathrm{~cm}^{2}$ molecule $\mathrm{s}^{-1}$, which indicates the potential of this more economical, laboratorybased technique to be employed in parallel with NR. In this case, temporal fluctuations in the optical signal are attributed to the mobility of islands of reaction products. We outline how such information may provide critical missing information in the identification of transient reaction products in a range of atmospheric surface reactions in the future.
\end{abstract}

Keywords: aerosol surface, kinetics, atmospheric reactions, air-water interface, methyl oleate, ozone, oxidation, neutron reflectometry, ellipsometry.

\section{Introduction}

Organic monolayers at the air-water interface have been widely studied because of their natural abundance, ${ }^{1,2}$ and their relevance for modification of the physical properties of formulations in common use. ${ }^{3,4}$ Our interest is the reaction kinetics of atmospheric relevance occurring at the surface of aerosol droplets. The reaction involves an insoluble organic monolayer and gas-phase oxidants. In the atmosphere, organic compounds are mainly oxidized by nitrate radicals, $\mathrm{NO}_{3}$, hydroxyl radicals, $\mathrm{OH}$ and ozone, $\mathrm{O}_{3}$. Knowledge of the kinetic parameters for monolayer oxidation is necessary for model studies aiming to deconvolute surface and bulk processes of atmospheric aerosols. ${ }^{5,6}$

A powerful technique used to study organic monolayers at the air-water interface is neutron reflectometry (NR). ${ }^{7}$ Neutrons are non-destructive for soft and biological materials, and by exploiting the method of isotopic substitution, i.e. deuteration of the monolayer as well as partial deuteration of the aqueous subphase to match its scattering properties to that of air, the technique can be used to quantify directly the surface excess during oxidation reactions. For example, a decrease in the surface excess during the reaction occurs when double bonds in unsaturated molecules are cleaved by the oxidant resulting in smaller reaction products with higher solubility and/or volatility. ${ }^{\text {e.g.8 }}$ As such, it is the rate of loss of material, which determines the evaporation rate of the subphase of key atmospheric relevance, which is measured.

NR has been applied previously to the atmospheric oxidation of organic monolayers in large custom-built reaction chambers with gas volumes on the order of 251 that contained a 
commercial Langmuir trough. ${ }^{8-11}$ The macroscopic air-water interface acted as a proxy for the surface of aerosol droplets. The large volume of the chamber placed an upper limit on the fastest reaction rates that could be measured due to uncertainties both in the time between introducing the oxidant flow and reaching steady state concentrations and in the nonhomogeneous gas diffusion. These issues did not limit the experiments because the time resolution of the technique used was typically on the order of several minutes. ${ }^{8,9}$ However, with the advent of new instrumentation, quantitative measurements of the surface excess of organic monolayers at the air-water interface can now be carried out with a time resolution as short as $1 \mathrm{~s} .^{10,11}$ The limitation in the efficiency of the experiments thus became the reaction chamber. There was therefore scope for significant improvements in the design of the chamber if its size could be reduced considerably without compromising the gas diffusion conditions as a result of physical perturbations to the free liquid surface.

Ellipsometry is a precise and sensitive optical reflectometry technique. ${ }^{12}$ It can, in principle, be used also to measure the surface excess during oxidation reactions at the air-water interface, but to our knowledge such measurements have not been published to date. The technique has the advantage that it is more accessible than NR as it can be carried out in a laboratory rather than a large-scale facility. Such an approach for the characterisation of single-component systems has the advantage that precious neutron beam time may be conserved for experiments which exploit selective deuteration of specific components in mixtures to determine relative reaction rates or partial deuteration of portions of a molecule to determine the reaction mechanism. Also, there is the potential to gain additional information concerning the lateral homogeneity of the interface during reactions given that temporal fluctuations in the optical signal reveal macroscopic domains of material present in a different phase. $^{13,14}$ Possible limitations, however, are that ellipsometry does not provide a direct quantification of the surface excess as the data need to be calibrated independently, and the measured optical signal would be affected by any changes in anisotropy in the interfacial layer during the reactions. To see if such complications could be overcome, the shapes of surface excess decays measured using the two techniques during the reactions would need to be compared. Such work would clearly be best carried out using the same reaction chamber to minimize uncertainties related to different gas mixing conditions. However, a reaction chamber compatible with both techniques has not been previously available.

To examine the complementary use of NR and ellipsometry in the study of atmospheric reactions at the air-water interface, we have developed a miniaturised reaction chamber with optimised conditions, i.e. a low volume for controlled gas injection and compatibility for equivalent measurements using the two techniques. To validate the performance of the chamber, we have studied the oxidation of methyl oleate monolayers by gas-phase ozone. Reasons for this choice of system are related to its high interest given that methyl oleate is both a key component of biodiesels ${ }^{15}$ and a food lipid that contributes to meat cooking emissions. ${ }^{16}$ An additional reason is that recently we reported a second order rate coefficient for methyl oleate ozonolysis of $(5.7 \pm 0.9) \times 10^{-10} \mathrm{~cm}^{2}$ molecule $\mathrm{s}^{-1} \mathrm{~s}^{-1}$ for the loss of material from the interface using NR on a large reaction chamber, ${ }^{10}$ thus providing a suitable reference. The scope of this study is to describe the design of the new chamber, to examine the potential resulting from the advanced instrumentation and new chamber to study faster oxidation reactions than were previously possible, and to explore the prospects of using ellipsometry in the study of single-component systems in the future to focus the use of neutrons in more studies of more complex systems. 


\section{Materials and Methods}

\subsection{Materials}

The organic monolayer comprised either deuterated methyl oleate $(d \mathrm{MO}$, Oxford Deuteration Facility, 95\%; see Pfrang et al. for sample characterisation) ${ }^{10}$ for NR or hydrogenous methyl oleate ( $h \mathrm{MO}$, Sigma-Aldrich, $\geq 99 \%$ ) for ellipsometry. The subphase was either a mixture of $8.1 \%$ by volume $\mathrm{D}_{2} \mathrm{O}$ (Eur-isotop, France) in pure $\mathrm{H}_{2} \mathrm{O}$ (generated using a Millipore purification unit, $18.2 \mathrm{M} \Omega \mathrm{cm}$ ), known as air contrast matched water (ACMW), for $\mathrm{NR}$ or pure $\mathrm{H}_{2} \mathrm{O}$ for ellipsometry. Chloroform (Sigma-Aldrich, $>99.8 \%$ ) and $\mathrm{O}_{2}$ (Air Liquide, France, $>99.9 \%$ ) were used as supplied.

\subsection{Gas setup}

$\mathrm{O}_{3}$ was produced by flowing pure $\mathrm{O}_{2}$ through a commercial ozoniser (UVP Pen-Ray continuous flow generator, UK). The $\mathrm{O}_{3}$ concentration was regulated by changing the flow rate and the exposure to the UV lamp. A flow of $\mathrm{O}_{3}$ in $\mathrm{O}_{2}$ was then admitted to the reaction chamber and the organic monolayer was oxidised at a rate that was determined by the $\mathrm{O}_{3}$ concentration. Measurements of $\mathrm{O}_{3}$ absorption at $253.7 \mathrm{~nm}$ were carried out using UV-visible spectroscopy to establish the volume concentration, $\left[\mathrm{O}_{3}\right]_{\mathrm{v}}$ (in molecule $\mathrm{cm}^{-3}$ ), and its uncertainty. ${ }^{17}$ The surface concentration, $\left[\mathrm{O}_{3}\right]_{\mathrm{s}}$ (in molecule $\mathrm{cm}^{-2}$ ), of ozone that dissolves into the organic layer at the air-water interface is calculated from $\left[\mathrm{O}_{3}\right]_{\mathrm{v}}$ assuming that the surface concentration is constant in time and is equal to Henry's Law solubility following the approach of Smith et al. ${ }^{18}$ (compare Pfrang et al.). ${ }^{10}$

\subsection{Neutron reflectometry}

A brief description of the physical basis of NR with reference to its application here can be found in part 1 and an example of the raw data can be found in part 2 of the Electronic Supporting Information. NR measurements of the oxidation of $d \mathrm{MO}$ monolayers by $\mathrm{O}_{3}$ in the new reaction chamber were carried out on FIGARO at the Institut Laue-Langevin (Grenoble, France). ${ }^{11}$ High neutron flux settings were used to maximise the data acquisition rate involving an incident angle of $0.62^{\circ}$, a wavelength range of $2-20 \AA$ (data reduction was carried out over the range of $3.4-20 \AA$ ), and a constant resolution in momentum transfer of $11 \%$. Normalisation of the reflectivity data was carried out with respect to the total reflection of an air- $\mathrm{D}_{2} \mathrm{O}$ measurement. The sample stage was equipped with passive and active antivibration control. The new reaction chamber was mounted on the sample stage, it was interfaced with the gas setup, and the custom-made miniature PTFE trough was filled with 80 $\mathrm{ml}$ of $\mathrm{D}_{2} \mathrm{O}$ for the optimisation of the gas mixing conditions (section 3.2) or of ACMW for the kinetic experiments (section 3.3). In the latter case, a monolayer with a surface concentration of $2.7 \times 10^{18}$ molecule $\mathrm{m}^{-2}$ was spread using $31.5 \mu \mathrm{lof} 1.13 \mathrm{mg} \mathrm{ml}^{-1} d \mathrm{MO}$ in chloroform. Data were recorded for a few minutes before $\mathrm{O}_{3}$ was admitted into the chamber at a flow rate of $51 \mathrm{~min}^{-1}$, leading to $\left[\mathrm{O}_{3}\right]_{\mathrm{s}}$ ranging from $(2.2 \pm 0.6) \times 10^{6}$ to $(4.0 \pm 0.9) \times 10^{7}$ molecule $\mathrm{cm}^{-2}$. The time resolution was $2 \mathrm{~s}$ except for an $\mathrm{O}_{2}$ blank recorded with a time resolution of $10 \mathrm{~s}$. The alignment of the interface was maintained using an optical sensor (LKG-152, Keyence, Japan), which operated through the laser alignment window. Data were analysed using the program MOTOFIT. ${ }^{19}$

\subsection{Ellipsometry}

A brief description of the physical basis of ellipsometry with reference to its application here can be found in part 3 and examples of the raw data with error bars can be found in part 4 of the Electronic Supporting Information. Ellipsometry measurements of the oxidation of $h \mathrm{MO}$ monolayers by $\mathrm{O}_{3}$ in the new reaction chamber were carried out using a phase modulated ellipsometer (Picometer Light Ellipsometer, Beaglehole Instruments, Wellington, NZ). The 
machine was equipped with a HeNe laser with a wavelength of $632.8 \mathrm{~nm}$ and the angle of incidence was $50^{\circ}$. The sample stage was equipped with active anti-vibration control. The new reaction chamber was mounted on the sample stage, it was interfaced with the gas setup, and the trough was filled with $80 \mathrm{ml}$ of $\mathrm{H}_{2} \mathrm{O}$. For the kinetic experiments (section 3.4), monolayers of $h \mathrm{MO}$ were prepared using an equivalent approach to that described above. Data were recorded for $250 \mathrm{~s}$ before $\mathrm{O}_{3}$ was admitted into the chamber at a flow rate of 1.81 $\min ^{-1}$, leading to $\left[\mathrm{O}_{3}\right]_{\mathrm{s}}$ in the ranging from $(9.0 \pm 0.6) \times 10^{6}$ to $(7.2 \pm 0.4) \times 10^{7}$ molecule $\mathrm{cm}^{-2}$. The time resolution was $5 \mathrm{~s}$. Adjustment of the liquid height during reactions was not required because the error introduced by evaporation was negligible.

The calibration between the measured optical phase shift, $\Delta$, and the surface excess, $\Gamma$, can be found in part 5 of the Electronic Supporting Information. In short, both for linear ${ }^{20}$ and empirical quadratic ${ }^{21}$ relations have been used in the literature for Gibb's monolayers. To our knowledge such a calibration has not been determined to date for Langmuir monolayers. Recently the importance of calibrating the relation on a case-by-case basis was emphasized. ${ }^{22}$ As such, we measured $\Delta$ using ellipsometry and $\Gamma$ using NR for equivalent spread amounts of $d \mathrm{MO}$ at the air-water interface and a linear relationship was determined.

\section{Results and Discussion}

\subsection{Chamber design}

A schematic cross section of the new reaction chamber is shown in Figure 1.a with a photograph of its exterior in Figure 1.b. The chamber is made of single blocks of aluminium due to its high strength, low neutron activation and low density. It weighs just $1.6 \mathrm{~kg}$, which is suitable for use on a range of active anti-vibration tables. A custom-made o-ring (omitted in Figure 1.a for clarity) between the top and bottom parts ensures efficient sealing. The internal volume of 0.91 is $\sim 30$ times lower than that of chambers used in previous related studies. A custom-made PTFE trough ( 13 wide $\times 10$ long $\mathrm{cm}^{2}$ ) is fixed at the bottom of the chamber by nylon screws. Sapphire windows for the neutron beam are rectangular $(90$ wide $\times 40$ high $\times 3$ thick $\mathrm{mm}^{3}$ ) and are positioned vertically. Quartz windows for the ellipsometry beam are circular (25 mm diameter) and are positioned in a tilted part of the lid at $50^{\circ}$ to the horizontal. Two o-rings for each window, one on each side, ensure gas-tight sealing as a result of their clamping by window frames fixed by stainless steel screws. The inlet and outlet ports for the gas flow are placed diametrically opposite on the short walls of the bottom part; the relative positions of inlet and outlet was chosen to optimise the homogeneity of the gas diffusion. The custom-made stainless steel gas connectors consist of a thin-walled stainless steel tube $(1 / 8$ inch outer diameter), and were inserted in and welded to a drilled hexagonal stainless steel screw. On the outer wall a $1 / 4$ inch tube is welded to the small tube, and on the inner part it is connected to a detachable PTFE tube (inner diameter $1 / 8$ inch), which is closed at the end and contains 11 holes drilled with a separation of $1 \mathrm{~cm}$ along the major axis.

\subsection{Gas injection}

Fluid dynamics simulations were performed to optimise the positioning of the gas connectors prior to the construction of the new reaction chamber. We used the ANSYS-CFX package (commercially available) based on finite volume analysis. In the simulation, $\mathrm{O}_{2}$ with a dynamic viscosity of $20.459 \mu \mathrm{Pa}$ s and a density of $1.2917 \mathrm{~kg} \mathrm{~m}^{-3}$ was used at $25{ }^{\circ} \mathrm{C}$. The inlet tube had an inner diameter of $3.27 \mathrm{~mm}$ and the linear speed was $10 \mathrm{~m} \mathrm{~s}^{-1}$, hence the flow rate was $51 \mathrm{~min}^{-1}$. These conditions resulted in a Reynolds number of 2064 at the entry of the inlet tube, which suggests that the model is close to the upper limit of laminar flow conditions at the max. flow of $51 \mathrm{~min}^{-1}$. We performed both laminar and turbulent calculations and observed negligible differences in the obtained velocities. The NR studies were performed at 
$51 \mathrm{~min}^{-1}$ while the ellipsometry experiments were performed at a flow rate of $1.81 \mathrm{~min}^{-1}$ i.e. well into the laminar flow regime. The line of holes is oriented in order to have initially a gas flow direction at $45^{\circ}$ with respect to the horizontal. Figure 2.a shows an example of a ray tracing image of the velocity resulting from the simulations under the optimum conditions: the $\mathrm{O}_{2}$ flow reaches the water surface with a low speed $\left(<0.5 \mathrm{~m} \mathrm{~s}^{-1}\right)$ and a pressure gradient of about $2 \times 10^{-3}$ mbar, hence the surface is only minimally affected by the gas flow.

Only fairly slow oxidation reactions of organic monolayers at the air-water interface involving the relatively mild oxidant $\mathrm{O}_{3}$ have been studied using NR to date. ${ }^{8-11}$ It would be useful also to be able to study faster reactions such as oxidation initiated by nitrate radicals, $\mathrm{NO}_{3}$ (typically $\sim 3$ orders of magnitude faster than equivalent reactions with $\mathrm{O}_{3}$ ) as well as hydroxyl radicals, $\mathrm{OH}$ (generally $\sim 3$ orders of magnitude more reactive than $\mathrm{NO}_{3}$ ). ${ }^{23}$ As such, we tested the maximum flow rates accessible in the new reaction chamber by examining the degree of perturbation on the liquid surface resulting from the gas flow. The tests were carried out by measuring the width of the specular peak of neutron reflection of an air $-\mathrm{D}_{2} \mathrm{O}$ measurement with respect to the flow rate, where broadening of the peak indicates the presence of surface waves. Figure 2.b shows the results for two different pieces of tubing, each $13-\mathrm{cm}$ long, used to diffuse the gas inside the chamber: tubing A had 11 holes of 1-mm diameter and delivers the gas more homogeneously at low flow rates and tubing B had 11 holes of 2-mm diameter which delivers the gas less turbulently at high flow rates. At low gas flow rates the detected full width half maximum peak width remains at its minimum value of $2.8 \mathrm{~mm}$, which shows that the gas flow does not produce measurable surface waves. This minimum value, determined by the collimating slit openings and the intrinsic detector resolution, is maintained to within a maximum broadening of $30 \%$ until the flow rate exceeds $\sim 51 \mathrm{~min}^{-1}$. This result is in keeping with the simulations discussed above.

A flow rate of $\sim 51 \mathrm{~min}^{-1}$ in the new reaction chamber leads to a significant reduction in the minimum mixing time: $\sim 10 \mathrm{~s}$ compared to at least $\sim 90 \mathrm{~s}$ previously. ${ }^{10}$ This improvement demonstrates the capability of the new chamber to be used to study faster reactions, e.g. more highly oxidised organic species with multiple functional groups and/or more potent oxidants such as $\mathrm{NO}_{3}$ uptake on organic surfaces. ${ }^{24}$ Information on the kinetics of such systems has not previously been accessible from NR studies at the air-water interface.

\subsection{Neutron reflectometry}

Recently we reported the second order rate coefficient for the oxidation of methyl oleate monolayers at the air-water interface by $\mathrm{O}_{3}$ as $(5.7 \pm 0.9) \times 10^{-10} \mathrm{~cm}^{2}$ molecule $\mathrm{s}^{-1}$. ${ }^{10}$ The measurements were carried out also using NR but in a much larger reaction chamber where it was more challenging to control and characterise the gas injection. In order to demonstrate the performance of the new reaction chamber, we recorded four surface excess decays with $\left[\mathrm{O}_{3}\right]_{\mathrm{s}}$ ranging from $2.2 \times 10^{6}$ to $4.0 \times 10^{7}$ molecule $\mathrm{cm}^{-2}$, while maintaining an optimum $\mathrm{O}_{2}$ flow rate of $51 \mathrm{~min}^{-1}$. Figure 3 shows the first order rate coefficients, $k_{1}=k\left[\mathrm{O}_{3}\right]_{\mathrm{s}}$, as a function of $\left[\mathrm{O}_{3}\right]_{\mathrm{s}}$ for data recorded in the new chamber in comparison with those reported previously from the old chamber. ${ }^{10}$ The new data are compatible with those previously reported as they fall well within the confidence limits. Furthermore, there are new data points at higher values of $\left[\mathrm{O}_{3}\right]_{\mathrm{s}}$ and they have statistically significantly smaller error bars. The solid line in Figure 3 corresponds to an orthogonal distance regression fit weighted by the uncertainties both in $k_{1}$ and $\left[\mathrm{O}_{3}\right]_{\mathrm{s}}$ using only the four runs performed with the new miniature reaction chamber. The resulting second order rate coefficient, $(5.4 \pm 0.6) \times 10^{-10} \mathrm{~cm}^{2}$ molecule ${ }^{-1} \mathrm{~s}^{-1}$, not only agrees with the recently determined value but is better constrained despite having used only 4 rather than 11 experimental runs. We have therefore demonstrated the potential to measure second 
order rate coefficients of atmospheric reactions of organic monolayers at the air-water interface (i) more precisely and (ii) involving faster reactions than was previously possible.

\subsection{Ellipsometry}

We recorded eight decays of the oxidation of $h \mathrm{MO}$ monolayers by $\mathrm{O}_{3}$ using ellipsometry with values of $\left[\mathrm{O}_{3}\right]_{\mathrm{S}}$ ranging from 0.9 to $7.2 \times 10^{7}$ molecule $\mathrm{cm}^{-2}$ while maintaining an $\mathrm{O}_{2}$ flow rate of $1.81 \mathrm{~min}^{-1}$. Figure 4 displays the resulting surface excess profiles with the error bars omitted for clarity (errors are given in Figure S2 in the supplementary information). The mean values of the ozone concentrations are reported in the legend of Fig. 4 and their uncertainties are in the range of $3.5-10 \%$.

The two slowest decays exhibit temporal fluctuations in the measured surface excess values. Such a feature in the data was also observed in refs 13 and 18 where it was attributed to the presence of macroscopic polymer/surfactant aggregates embedded in Gibb's monolayers. In the present case, however, the fluctuations are likely to originate instead from the presence of macroscopic islands of reaction products that are segregated from the reactant in the Langmuir monolayer due to de-mixing, and that have different optical properties. The optical signal fluctuates with time because the islands are transported in and out of the area probed by the laser $\left(\sim 1 \mathrm{~mm}^{2}\right)$ by Brownian motion and probably also Marangoni flow. (The question is raised of whether this process affects the NR data analysis but the change in scattering length density between the deuterated reactant and deuterated reaction products will be minimal.) The surface excess values fall to zero with time, which shows that these products are present only transiently at the interface before they evaporate or dissolve. The size of the islands remains unresolved because to date we have not managed to couple the new reaction chamber with a Brewster angle microscope during oxidation reactions (given the short focal distance of the microscope objective together with the required protection of the objective from the highly oxidising gas-phase environment) but it follows that they have a length scale up to the millimetre range. Incorporation of a protected Brewster angle microscope into a larger reaction chamber would benefit future studies even though the lateral morphologies observed would not be directly comparable with the kinetic data recorded using NR and ellipsometry in the miniaturised chamber due to the different gas mixing conditions. Brewster angle microscopy of $h \mathrm{MO}$ monolayers prior to the decay reactions has been carried out on an open trough, and the resulting images recorded at different surface pressures are presented in part 6 of the Electronic Supporting Information. Such fluctuations have not been revealed using NR which can be explained in terms of the much larger probed area of the interface $\left(\right.$ several $\left.\mathrm{cm}^{2}\right)$. The complementarity of using ellipsometry and NR to provide additional information for the identification of reaction products, which is not trivial due to the small quantities produced combined with the lack of stability at the interface, is therefore demonstrated. Also, X-ray reflectometry may be used as an alternative complementary technique that is compatible with our miniaturised reaction chamber (this would require only changes of the vertical windows of the chamber from sapphire to, e.g., kapton), and additional structural information (e.g. tilting angle $)^{25}$ may then be accessible thanks to the larger range of momentum transfer values accessible.

Figure 5 shows the values of $k_{1}$ from each fitted $\Gamma(t)$ profile as a function of $\left[\mathrm{O}_{3}\right]_{\mathrm{s}}$. The linear fit leads to the determination of a rate coefficient of $(5.0 \pm 0.9) \times 10^{-10} \mathrm{~cm}^{2}$ molecule $\mathrm{s}^{-1}$; the error reported is at $95 \%$ confidence limits. The fit was performed taking into account uncertainties in the $k_{1}$ values from ellipsometry and in the ozone concentrations. The negative intercept lies well within the uncertainty of the fit. It should be noted that the ellipsometry experiments were performed with $h \mathrm{MO}$ on pure water while the NR work was performed with $d \mathrm{MO}$ on $\mathrm{ACMW}$ (i.e. $8.1 \% \mathrm{D}_{2} \mathrm{O}$ in $\mathrm{H}_{2} \mathrm{O}$ ). Ozonolysis is known to involve radical species that 
could display a kinetic isotope effect, but our results suggest that there is no significant isotope effect on the kinetics of the methyl oleate ozonolysis. The rate coefficient determined here is in agreement with the values discussed above that were measured using NR. Ellipsometry has therefore proved to be an adequate substitute for NR in the study of the oxidation of methyl oleate monolayers by $\mathrm{O}_{3}$. Such a positive result must be reported with a degree of caution, however, as it is possible that different reaction products that have different optical properties to the reactant may be retained at the interface for longer in other systems, which could render the surface excess calibration and therefore the kinetic analysis inaccurate. We advise therefore that new systems are validated on a case-by-case basis, i.e. a limited number of measurements should be carried out using both techniques prior to the acquisition of a complete data set using only ellipsometry. Nevertheless the indication is promising that neutron beam time may be focussed more efficiently in the future towards studies of more complex systems.

\section{Conclusions \& Outlook}

We have discussed the strengths and limitations of the combined application of NR and ellipsometry in the study of atmospherically-relevant chemical reactions at the air-water interface. For this work we commissioned a miniaturised reaction chamber, the main features of which are a low volume for controlled gas injection and compatibility for equivalent measurements using the two techniques. The motivation behind this development was to keep abreast with advances in NR instrumentation so that more precise determinations of rate coefficients can be carried out and faster reactions can be studied. The rate coefficient of the oxidation of methyl oleate monolayers by $\mathrm{O}_{3}$ recorded using $\mathrm{NR}$ in the new chamber is consistent with that reported previously from samples measured in a large reaction chamber and is better constrained in spite of the fact that the analysis is based on fewer data points. This general improvement in performance opens up in the future the possibility to access the kinetics of chemical reactions that involve more reactive organic materials and/or more potent gas-phase oxidants.

Ellipsometry was also applied to measurements of the same system in the new chamber and a consistent rate coefficient was determined. The agreement between the data recorded using the two techniques validates our original hypothesis concerning the use ellipsometry to replace NR in the determination of the rate coefficient of the oxidation of single-component monolayers. Our results also suggest that there is no significant kinetic isotope effect for the ozonolysis of methyl oleate at the air-water interface. Nevertheless, for the slowest reactions studied ellipsometry revealed temporal fluctuations in the optical signal, which we attribute to the transient presence of segregated islands of reaction products with different optical properties. On the one hand, we believe that this complementary information may be useful in the future as a mean to help with the tricky identification of the reaction products, e.g. by tests of the mixing of the reactant with different possible products using Brewster angle microscopy. On the other hand, the results prompt us to apply a degree of caution in our conclusion about the use of ellipsometry as a substitute for NR in the stated application: if products with different optical properties were to remain at the interface for longer in different systems, and the calibration of the optical signal to the surface excess is different for the reactant and products, then the kinetic analysis may no longer be valid. Even though each new system needs to be validated on a case-by-case basis, we demonstrate the potential for neutron beam time to be conserved for more sophisticated studies such as those involving mixed monolayers (with selective isotopic substitution) and the determination of reaction mechanisms (with partial isotopic substitution).

\section{Acknowledgements}


We thank Alessio Laloni for the support with the design and construction of the new reaction chamber, Fredric Thomas for performing the fluid dynamics simulations, Bob Thomas and the Oxford Deuteration Facility for the provision of the deuterated methyl oleate, Kunal Rastogi and Francesco Piscitelli for help during neutron experiments, the Partnership for Soft Condensed Matter for access to the ellipsometer and the Brewster angle microscope, and the ILL (Grenoble, France) for funding the construction of the chamber and allocations of beam time on FIGARO. FS is grateful for support from the ILL and the University of Reading in the framework of the NEATNOx studentship. CP thanks NERC (grant number NE/G000883/1) for support.

\section{References}

1. Fuzzi, S., Andreae, M. O., Huebert, B. J., Kulmala, M., Bond, T. C., Boy, M., Doherty, S. J., Guenther, A., Kanakidou, M., Kawamura, K., Kerminen, V.-M., Lohmann, U., Russell, L. M., Pöschl, U. Critical assessment of the current state of scientific knowledge, terminology, and research needs concerning the role of organic aerosols in the atmosphere, climate, and global change. Atmos. Chem. Phys. 2006, 6, 2017-2038.

2. Perez-Gil, J. Structure of pulmonary surfactant membranes and films: The role of proteins and lipid-protein interactions. BBA-Biomembranes 2008, 1778, 1676-1695.

3. Fainerman, V., Mobius, D., Miller, R., 2001. Surfactants - Chemistry, Interfacial properties, applications. Elsevier science \& technology.

4. La Mesa, C. Polymer-surfactant and protein-surfactant interactions. J. Colloid Interface Sci. 2005, 286, 148-157.

5. Pfrang, C., Shiraiwa, M., Pöschl, U. Chemical ageing and transformation of diffusivity in semi-solid multi-component organic aerosol particles. Atmos. Chem. Phys. 2011, 11, 73437354.

6. Renbaum-Wolff, L., Grayson, J.W., Bateman, A. P., Kuwata, M., Sellier, M., Murray, B. J., Shilling, J. E., Martin, S. T., Bertram, A. K. Viscosity of $\alpha$-pinene secondary organic material and implications for particle growth and reactivity. P. Natl. Acad. Sci. USA 2013, 110, 80148019.

7. Lu, J., Thomas, R., Penfold, J. Surfactant layers at the air/water interface: structure and composition. Adv. Colloid Interface Sci. 2000, 84, 143-304.

8. King, M. D., Rennie, A. R., Thompson, K. C., Fisher, F. N., Dong, C. C., Thomas, R. K., Pfrang, C., Hughes, A. V. Oxidation of oleic acid at the air-water interface and its potential effects on cloud critical supersaturations. Phys. Chem. Chem. Phys. 2009, 11, 7699-7707.

9. King, M. D., Rennie, A. R., Pfrang, C., Hughes, A. V., Thompson, K. C. Interaction of nitrogen dioxide $\left(\mathrm{NO}_{2}\right)$ with a monolayer of oleic acid at the air-water interface - a simple proxy for atmospheric aerosol. Atmos. Environ. 2010, 44, 1822-1825.

10. Pfrang, C., Sebastiani, F., Lucas, C. O. M., King, M. D., Hoare, I. D., Chang, D., Campbell, R. A. Ozonolysis of methyl oleate monolayers at the air-water interface: oxidation kinetics, reaction products and atmospheric implications. Phys. Chem. Chem. Phys. 2014, 16, $13220-13228$.

11. Campbell, R. A., Wacklin, H. P., Sutton, I., Cubitt, R., Fragneto, G. Figaro: The new horizontal neutron reflectometer at the ILL. Eur. Phys. J. Plus 2011, 126, 107.

12. Azzam, R. M. A. Bashara N. M., 1997. Ellipsometry and polarised light. North-Holland Publishing Company. 
13. Péron, N.; Cagna, A.; Valade, M.; Bliard, C.; Aguié-Béghin, V.; Douillard, R. Layers of macromolecules at the champagne/air interface and the stability of champagne bubbles. Langmuir 2001, 17, 791-797.

14. Campbell, R. A.; Yanez Arteta, M.; Angus-Smyth, A.; Nylander, T.; Noskov, B. A.; Varga, I. Direct impact of non-equilibrium aggregates on the structure and morphology of Pdadmac/SDS layers at the air/water interface. Langmuir 2014, 30, 8664-8674.

15. Wang, Y., Cannon, F. S., Salama, M., Fonseca, D. A., Giese, S. Characterization of pyrolysis products from a biodiesel phenolic urethane binder. Environ. Sci. Technol. 2009, $43,1559-1564$.

16. Allan, J. D., Williams, P. I., Morgan, W. T., Martin, C. L., Flynn, M. J., Lee, J., Nemitz, E., Phillips, G. J., Gallagher, M. W., Coe, H. Contributions from transport, solid fuel burning and cooking to primary organic aerosols in two UK cities. Atmos. Chem. Phys. 2010, 10, $647-668$.

17. Mauersberger, K., Hanson, D., Barnes, J., Morton, J. Ozone vapor pressure and absorption cross-section measurements: Introduction of an ozone standard. J. Geophys. Res-Atmos. $1987,92,8480-8482$.

18. Smith, G. D., Woods, E., DeForest, C. L., Baer, T., Miller, R. E. Reactive uptake of ozone by oleic acid aerosol particles: application of single-particle mass spectrometry to heterogeneous reaction kinetics. J. Phys. Chem. A 2002, 106, 8085-8095.

19. Nelson, A. Co-refinement of multiple-contrast neutron/x-ray reflectivity data using MOTOFIT. J. Appl. Crystallogr. 2006, 39, 273-276.

20. Angus-Smyth, A., Bain, C. D., Varga, I., Campbell, R. A. Effects of bulk aggregation on $\mathrm{PEI} / \mathrm{SDS}$ monolayers at the dynamic air/liquid interface: depletion due to precipitation versus enrichment by a convection/spreading mechanism. Soft Matter 2013, 26, 6103-6117.

21. Manning-Benson, S., Bain, C. D., Darton, R. C. Measurement of dynamic interfacial properties in an overflowing cylinder by ellipsometry. J. Colloid Interface Sci. 1997, 189, $109-116$.

22. Li, P. X., Thomas, R. K., Penfold, J. Limitations in the use of surface tension and the Gibbs equation to determine surface excesses of cationic surfactants. Langmuir 2014, 30, 6739-6747.

23. NIST Kinetic Database, 2013. http://kinetics.nist.gov/kinetics (accessed 07/09/2014).

24. Gross, S. and Bertram, A. Reactive uptake of $\mathrm{NO}_{3}, \mathrm{~N}_{2} \mathrm{O}_{5}, \mathrm{NO}_{2}, \mathrm{HNO}_{3}$, and $\mathrm{O}_{3}$ on three types of polycyclic aromatic hydrocarbon surfaces. J. Phys. Chem. A 2008, 112, 3104-3113.

25. Konovalov, O. V \& Vorobiev, A. A. Fast acquisition of extensive X-ray diffraction patterns of a gas-liquid interface in grazing-incidence geometry. J. Appl. Crystallogr. 2013, $46,270-275$. 

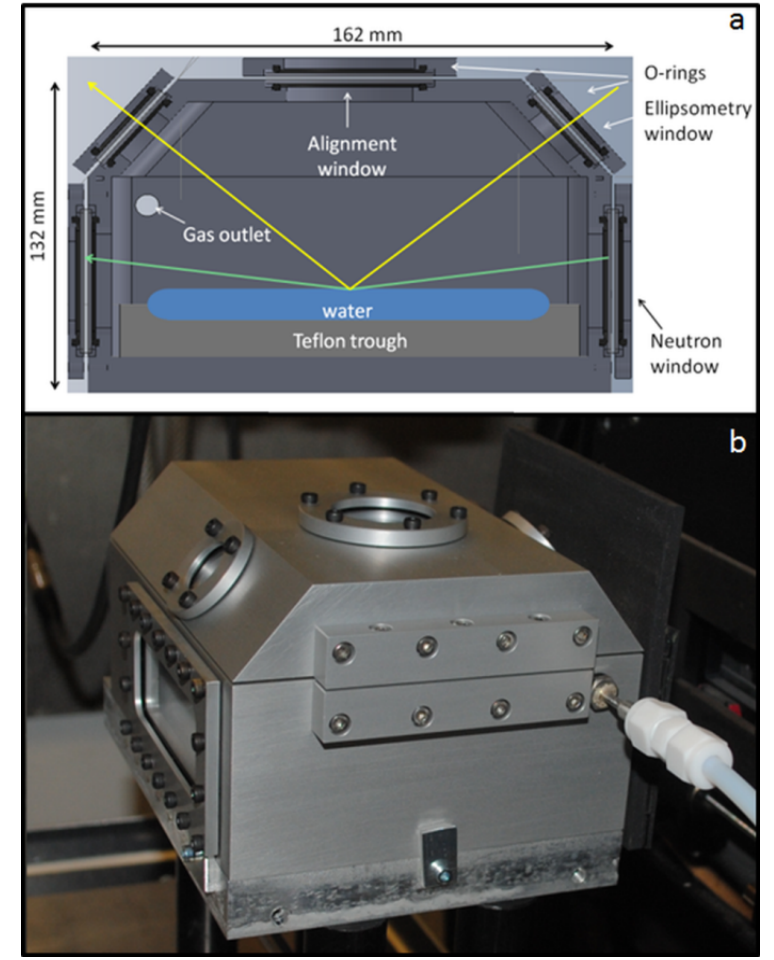

3 Figure 1: a. Schematic cross section of the 4 reaction chamber. The green arrow 5 indicates the path of the neutron beam and 6 the yellow arrow indicates the path of the 7 laser beam for ellipsometry. The angle of 8 incidence of the neutrons has been 9 exaggerated for clarity. b. Photograph of 10 the reaction chamber installed on the 11 FIGARO sample stage. The neutron exit 12 window, the laser alignment window (top), 13 the ellipsometry windows and the gas inlet 14 can be seen.

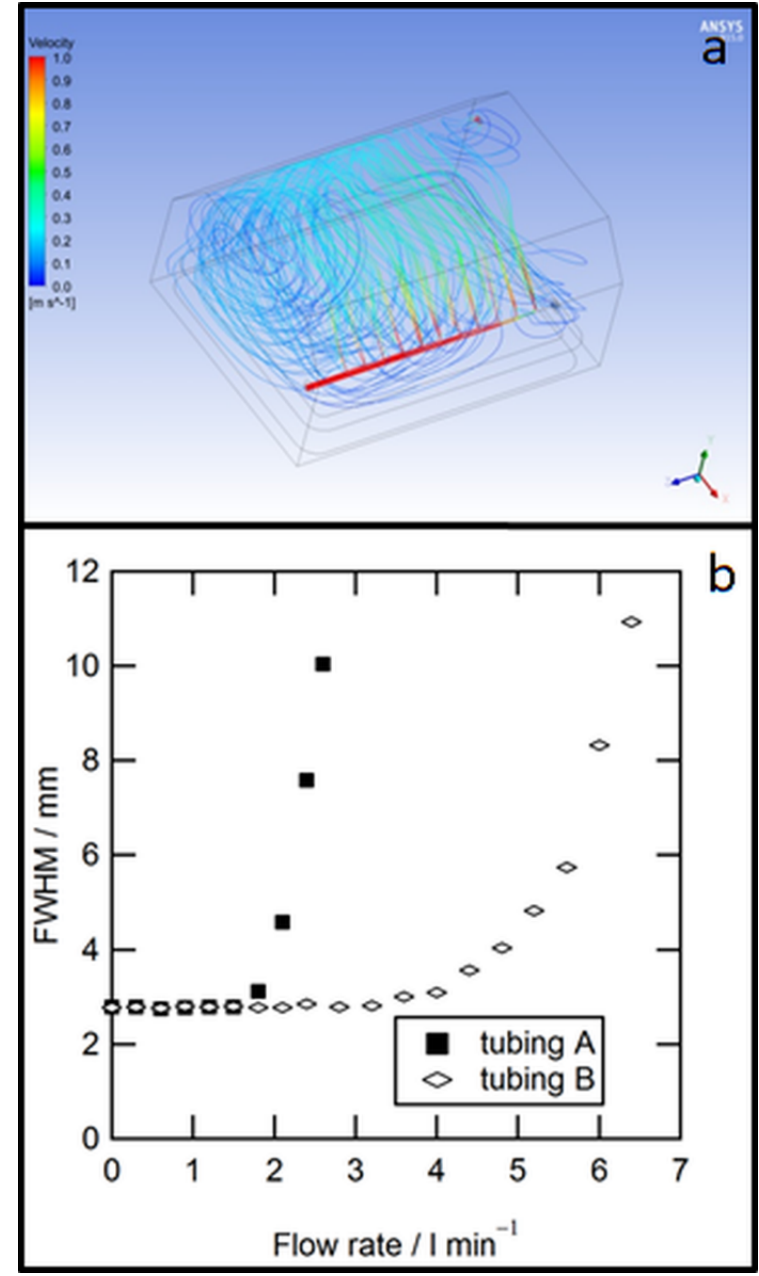

19 Figure 2: a. Ray tracing image of the 20 velocity profile in the reaction chamber 21 when simulating a flow of pure $\mathrm{O}_{2}$ at $25^{\circ} \mathrm{C}$ 22 and $51 \mathrm{~min}^{-1}$. b. Width of the peak 23 corresponding to the specular reflection of 24 neutrons at a clean air- $\mathrm{D}_{2} \mathrm{O}$ interface with 25 respect to the flow rate through the reaction 26 chamber. Two different inlet systems were 27 employed: tubing A with 11 holes of 1-mm 28 diameter (black squares) and tubing $\mathrm{B}$ with 2911 holes at 2-mm diameter (white 30 diamonds). Tubing A was used at a flow 31 rate of $1.81 \mathrm{~min}^{-1}$ for all the ellipsometry 32 studies while tubing B was used at the max. 33 flow rate of $51 \mathrm{~min}^{-1}$ for NR. 


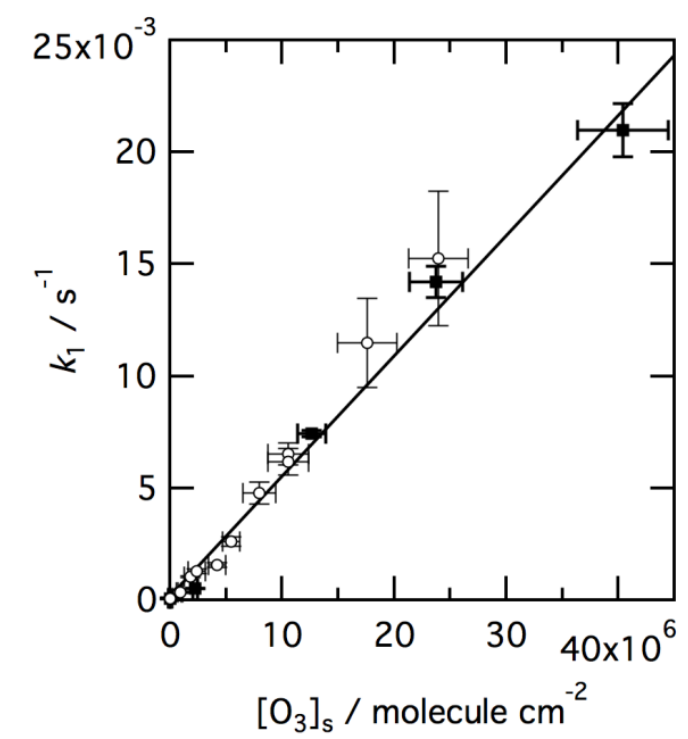

2 Figure 3: Pseudo-first order rate 3 coefficients, $k_{1}$, as a function of the ozone 4 surface concentration, $\left[\mathrm{O}_{3}\right]_{\mathrm{s}}$, for methyl 5 oleate monolayers measured using NR in 6 the new reaction chamber (black squares) 7 and those reported previously in a large 8 chamber (white circles). ${ }^{10}$ The error bars 9 represent the associated uncertainties at one 10 standard deviation. The solid line 11 corresponds to an orthogonal distance 12 regression fit weighted by the uncertainties 13 both in $k_{1}$ and $\left[\mathrm{O}_{3}\right]_{\mathrm{s}}$ using only the four new 14 data points. The small positive intercept lies 15 well within the uncertainty of the fit $((2 \pm$ $\left.167) \times 10^{-4} \mathrm{~s}^{-1}\right)$. 


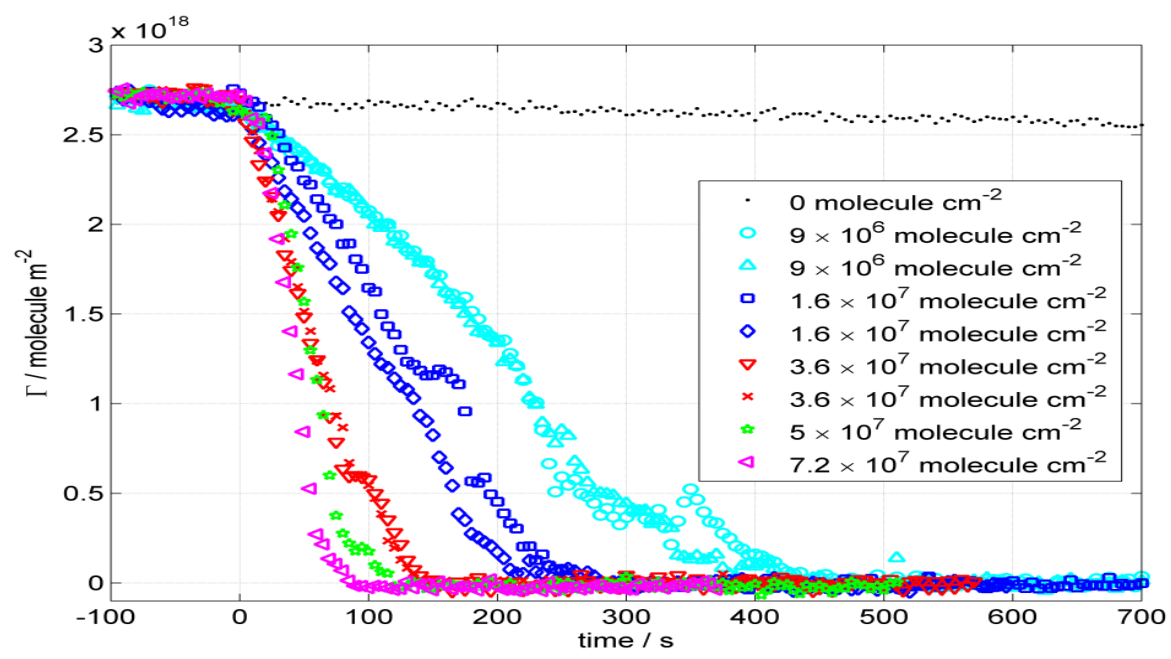

Figure 4: Surface excess decays as a function of time for $h \mathrm{MO}$ monolayers oxidised by $\mathrm{O}_{3}$ measured using ellipsometry. The $\mathrm{O}_{2}$ flow rate was fixed to $1.81 \mathrm{~min}^{-1}$ and the setting of the ozoniser was varied. The legend displays the mean values of the ozone surface concentration, $\left[\mathrm{O}_{3}\right]_{\mathrm{s}}$. The error bars are omitted for visual clarity; the errors range between 3.5 and $10 \%$ (the errors increase as the surface excess decreases in each experimental run). The equivalent plot with error bars can be found in part 4 of the Electronic Supporting Information.

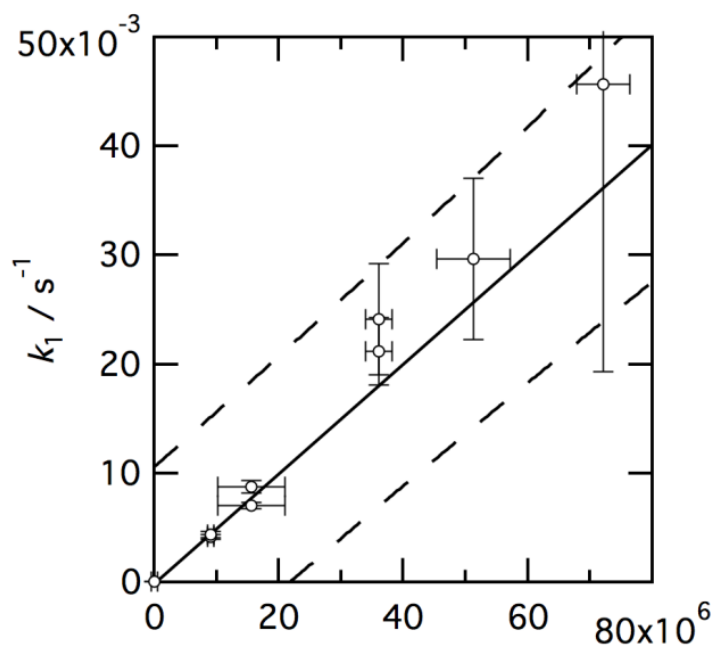

$\left[\mathrm{O}_{3}\right]_{\mathrm{s}} /$ molecule $\mathrm{cm}^{-2}$

Figure 5: Pseudo-first order rate coefficients, $k_{1}$, as a function of the ozone surface concentration, $\left[\mathrm{O}_{3}\right]_{\mathrm{s}}$, for methyl oleate monolayers measured using ellipsometry in the new reaction chamber (white circles). The error bars represent the associated uncertainties at one standard deviation. The solid line corresponds to an orthogonal distance regression fit weighted by the uncertainties both in $k_{1}$ and $\left[\mathrm{O}_{3}\right]_{\mathrm{s}}$. The small negative intercept lies well within the uncertainty of the fit $\left(-(1 \pm 6) \times 10^{-4} \mathrm{~s}^{-1}\right)$. The dashed lines represent the $95 \%$ prediction bands. 